\title{
Bio-mimetic Finger Human like morphology, control \& motion planning for intelligent robot \& prosthesis
}

\author{
Emanuele Lindo Secco, Giovanni Magenes \\ University of Pavia, Bio-Engineering Laboratory - DIS - Italy \\ Emanuele@bioing.unipv.it, Giovanni@bioing.unipv.it
}

\section{Introduction}

The aim of this hapter is to summarize results we have obtained over the past five years of the bio-mimetic approach to robotics and/or prosthetic applications .

Focusing on multiple degrees of freedom (dof) and multi-articulated limbs we will suggest technical solutions for controlling and planning the motion of an artificial limb in such a way that it will match the natural pattern of 'natural movement'.

We considered three items when regarding the problem of moving a 3 dof artificial finger:

- First, we describe the morphology of an ideal bionic finger through imitation of the anatomical shape of the human finger as well as optimization criteria of the grasping function;

Second, we introduce a mathematical model of multi-articular human limbs suggesting a solution to the problem of acting multiple-links with unimodal bell shape speed profiles;

Third, we propose a natural planning of finger kinematics and dynamics. The plan will be based on desired and proprioceptive signals driving from a neural network $(n n)$ controller: after a learning phase, the 'intelligent' outcome (AI system) will be able to reproduce the effective dynamics of natural movement. Furthermore the $n n$ exhibits the capacity to generate 'never before seen' movements.

Future perspectives on control strategies will be discussed.

Abbreviations

neural network $\quad \mathrm{Nn}$

degree of freedom Dof

\section{Bio-mimetic Morphology}

\subsection{Introduction}

The design of an artificial finger, specifically the design of the three phalanges of an artificial finger, depends on its aim. In this work we have simplified the representation of the intersegments by modeling the phalanges with cylindrical rods,. The main goal is to control 
movement and not to grasp an object, which implies more complex concepts of contact mechanics. This approach simplifies the computation of the dynamic equations. Other authors have preferred different approaches, for example grasping analysis or modeling, in which the shape of the phalanges is more important.

In the design of an artificial finger we must first fix the dimensions of each phalanx. We started by considering a Fibonacci series, as the basis for computing the right proportional structure of the finger (Gupta et al., 1998). We also consulted the ergonomics literature of Garrett, 1971, Buchholz et al., 1992 and Pheasant, 1996. After fixing the lengths, we developed the shape.

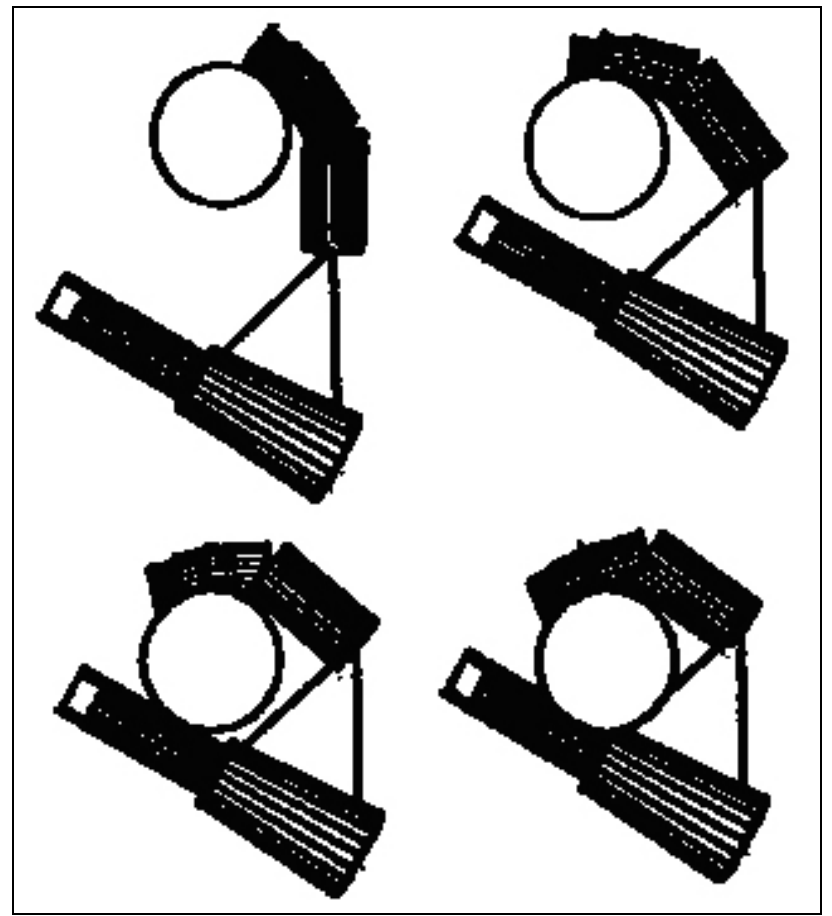

Fig. 1. The model proposed by Esteki \& Mansour, 1997, includes Thumb, Index Finger, Palm and Inter-segmental Joints. In the sequence of the images, the cylinder is successfully grasped between the index finger, thumb and palm. Initial, final and two intermediate pictures of the hand motion are presented (figure from the cited paper).

\subsection{Some Examples}

We considered the work of Esteki \& Mansour (1997) to model hand positioning and grasping force. They treat the hand as a jointed multi-body system. Thumb, Index Finger, Palm and Inter-segmental Joints are included in the model (Fig. 1). Each phalanx is represented by a cylindrical rigid body whose dimensions are measured for one subject. Segment masses and principal moments of inertia are computed on the basis of the volume and geometry of each segment, using a specific mass of $1.1 \mathrm{~g} / \mathrm{cm}^{3}$. Moreover, it is assumed that the shape of the contacting thumb surface is a section of a sphere and that only the distal phalanx of the thumb is in contact with the object. 


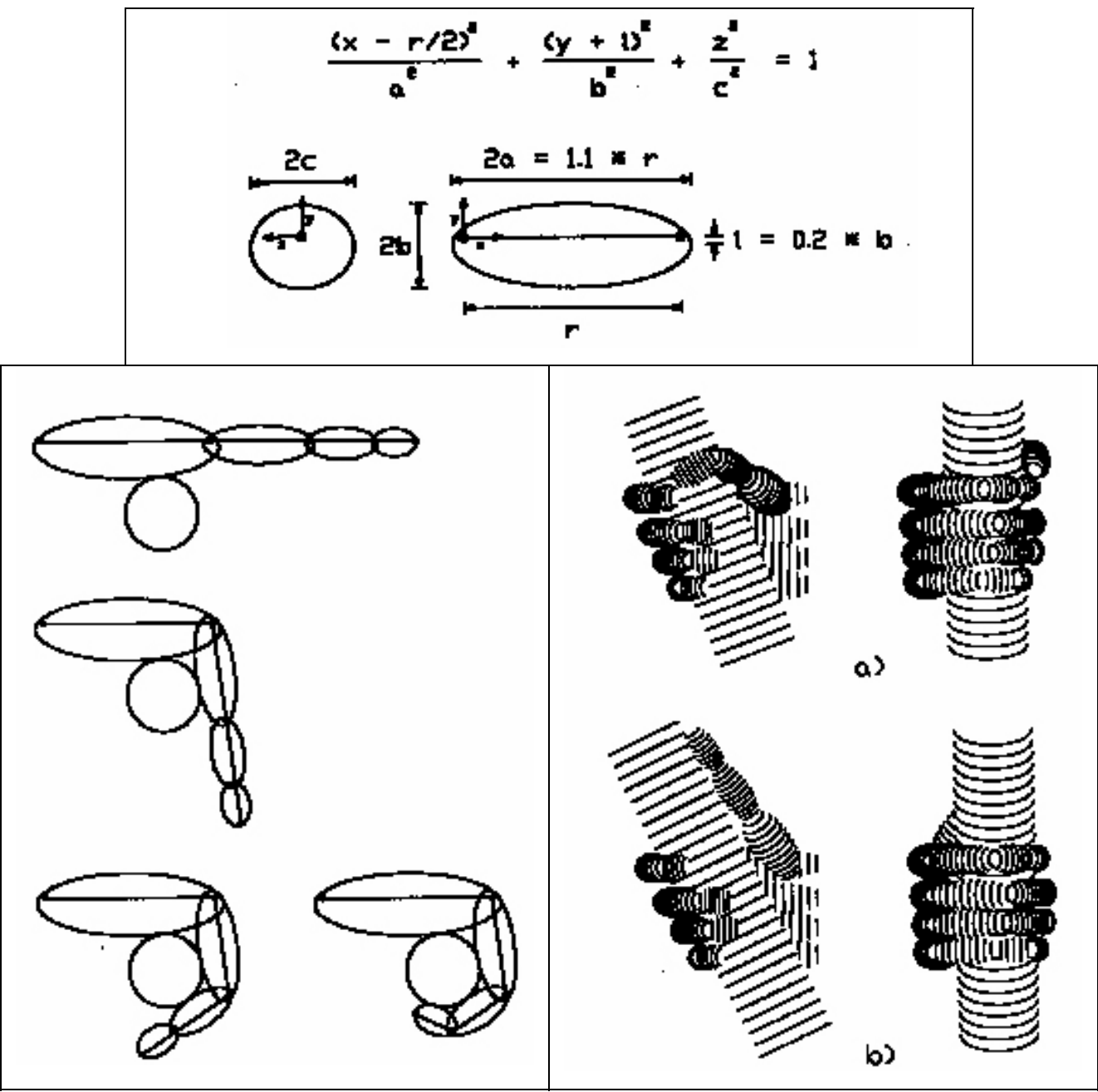

Fig. 2. TOP PANEL. Equations and graphical description of the three-dimensional geometry of the hand ellipsoidal segments; $r=$ kinematic segment length, $a=$ length semi-axis dimension, $\mathrm{b}=$ depth semi-axis dimension, $\mathrm{c}=$ breadth semi-axis dimension, 1 = ellipsoid depth offset. BOTTOM LEFT PANEL. Planar example of the power grasp algorithm from Buchholz \& Armstrong, 1992; BOTTOM RIGHT PANEL. Example of the graphical display capabilities of the model: different view of the (a) transverse volar grasp and (b) diagonal volar grasp are shown (images from the cited paper).

Another interesting kinematical model was developed by Buchholz \& Armstrong (1992) to simulate and predict the prehensile abilities of the human hand. The model is based on an algorithm that determines the contact between two ellipsoids (Fig. 2), which are used to approximate the geometry of the cutaneous surface of the hand segments. 


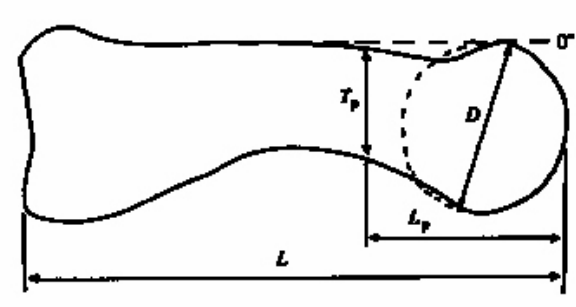

(a) Sagiton plux

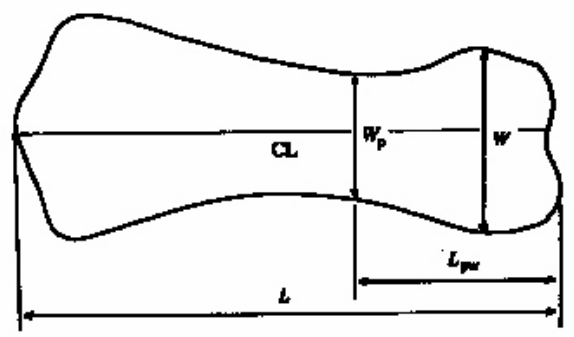

(b) Frintil plune

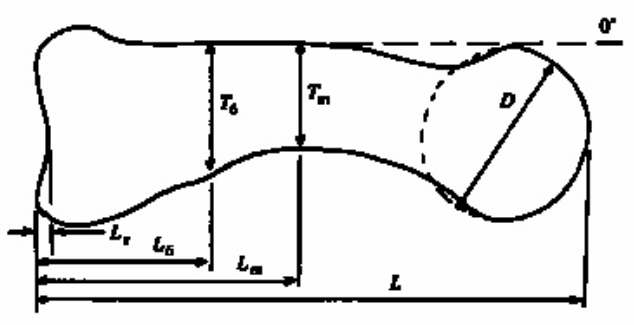

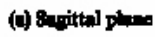

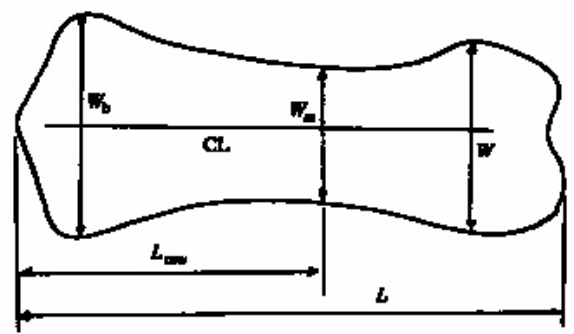

(b) Fontal pinos

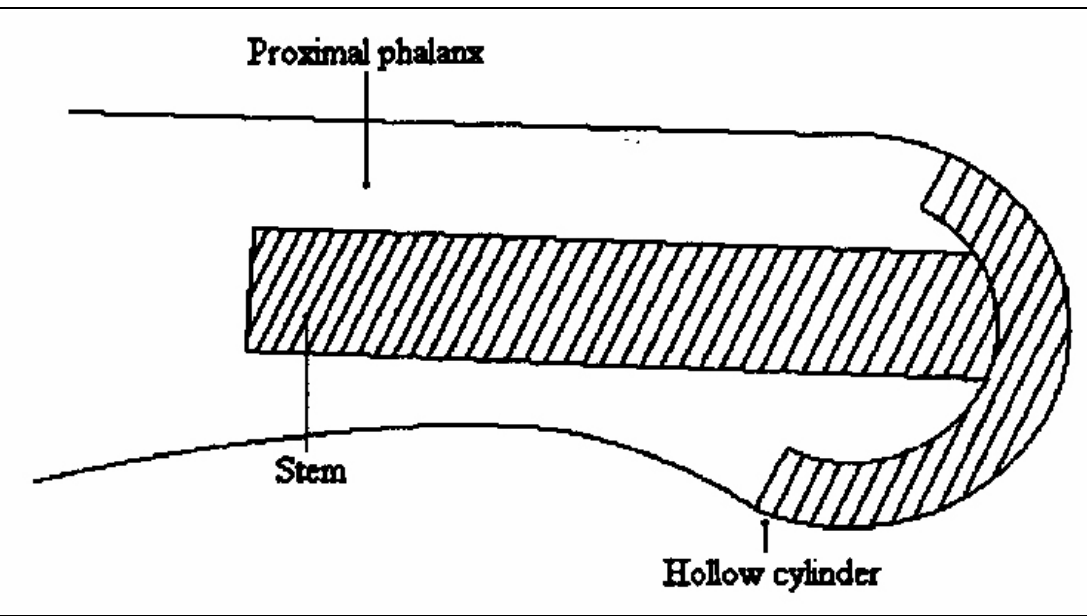

Fig. 3. TOP PANEL. MetacarpoPhalangeal (MP) dimensions and ( $\mathrm{dx}$ ) Proximal InterPhalangeal (PIP) dimensional parameters (imagine from). BOTTOM PANEL. Schema of a hollow PIP joint surface replacement prosthesis (images from Ash \& Unsworth, 1996 \& Ash et al., 2000).

Coefficients to estimate anthropometric parameters from hand length and breadth are incorporated in this model. The model was developed to predict or quantify the grip posture (Fig. 2, bottom panel). Moroever Buchholz collected data that would allow modeling the surface of each segment as a group of ellipsoids instead of a single ellipsoid. 
Another approach to design artificial finger or portions of the finger came from the medical environment: phalanges bones from 83 PIP joints were dissected in order to determine the shape and size of the articular surface (Ash \& Unsworth, 1996). Then, the dimensions were used in the design of surface replacement prosthesis for the PIP joint. Finally, from a statistical analysis of the parameters (Fig. 3, top panel), a cross-linked polyethylene (XLPE) surface replacement finger joint prosthesis (Fig. 3, bottom panel) was designed (Ash \& Unsworth, 2000).

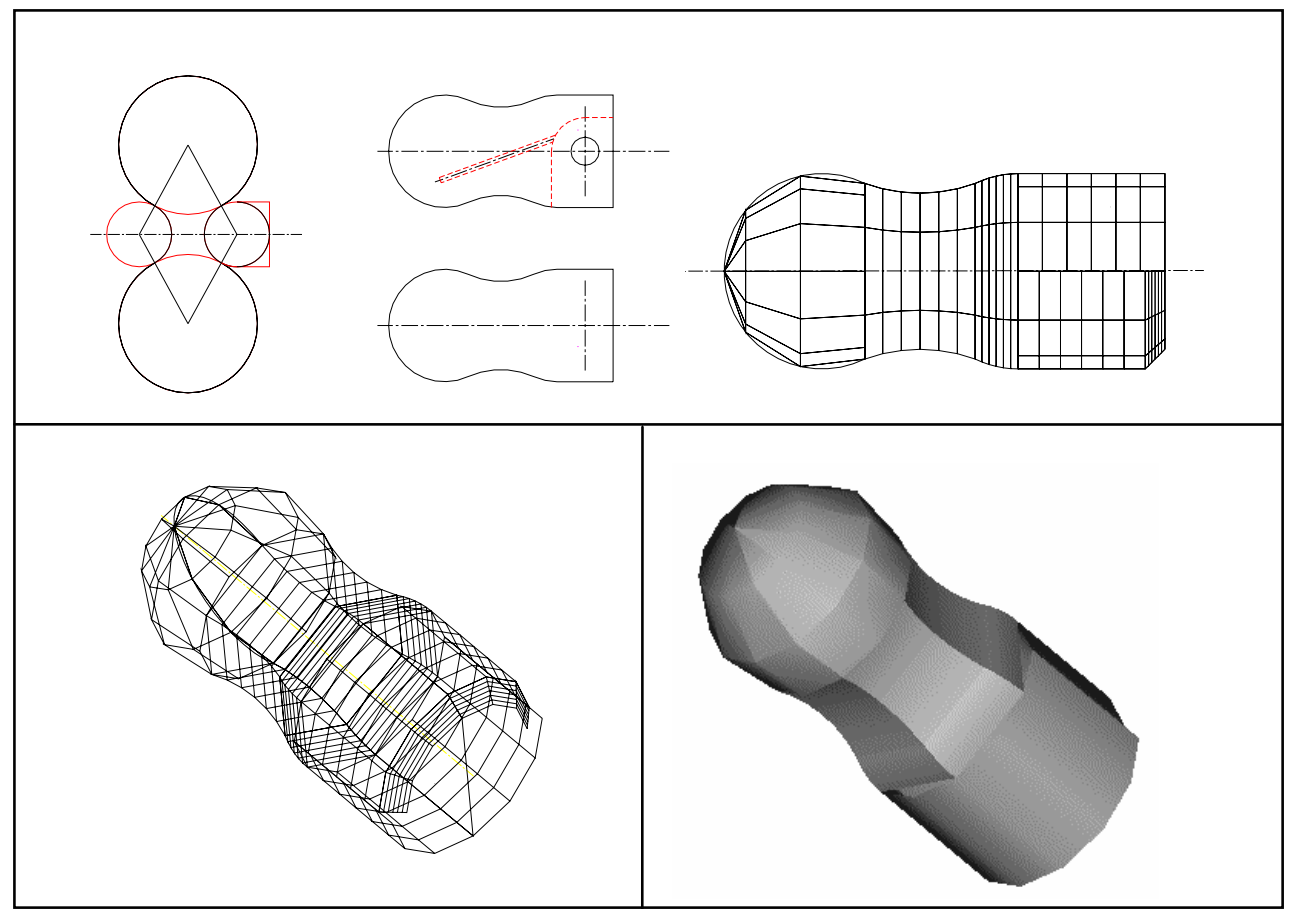

Fig. 4. TOP PANEL. Details on the rational design of the DIP phalanx for the artificial middle finger prosthesis. BOTTOM PANEL. The 3-D mesh (left) and rendering (right). Dimension is similar to the natural one (images from Secco, 1997).

PIP joint replacement is just a little portion of the entire finger and of the entire hand, whereas many attempts have been made by many laboratories for realizing artificial fingers and/or entire robotic or prosthetic hands in the last 10-20 years (for a rapid review see Rosheim, 1994).

In this context, we would like to introduce some proposals for the realization of a 3 dof robotic middle finger (Secco, 1997). The profiles of the phalanges imitate the human finger phalanges (Fig. 4), while the movement is guaranteed by the action of three wires (like human tendons) into the mechanical structure. Flexion is supported by 3 active tendons, extension is effected by pre-compressed springs into the three joints (Fig. 5). 

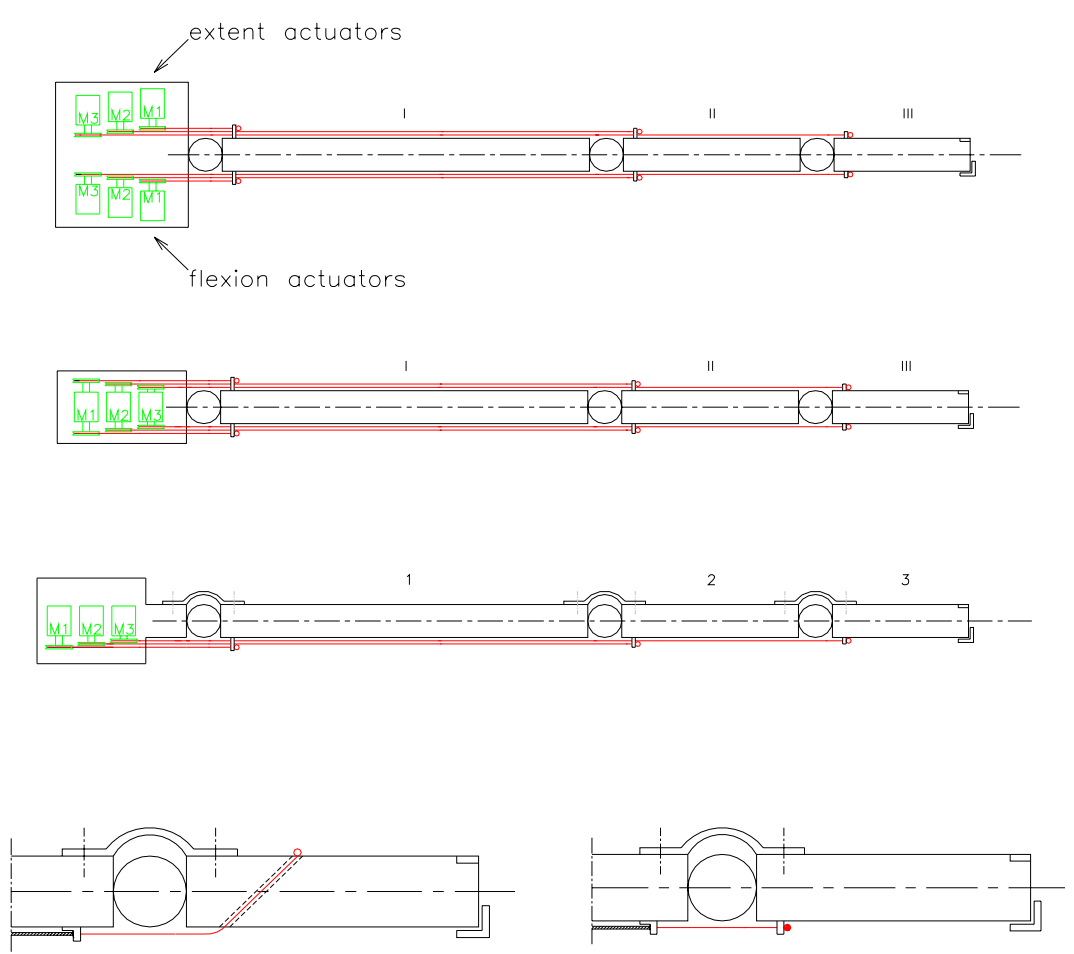

Fig. 5. Three potential motor configurations. TOP PANEL. Extent and flexion actuators (i.e. 6 motors for 3 independent phalanges). This configuration is expensive in terms of number of actuators and of mechanical complexity - adopted by the MIT Hand, i.e. 266 pulleys to drive all the tendons network - Jacobsen et al., 1986. MIDDLE PANEL. Extent \& flexion are driven by the same motor for each phalanx. In this configuration the tendon excursions (flexion and extension) must be the same - this configuration is adopted, for example, by the UB Hand II - Melchiorri \& Vassura, 1995). BOTTOM PANEL. Flexion is actively relized by motors, while extent follows by the spring responses.BELOW. Details over the tendon attachment on the third DIP phalanx.

Different motor configurations were analyzed to establish if flexion and extension should be activated by 3 (or 6) independent motors and/or by passive springs placed around each articulations (Fig. 5). Tendons attached to the finger's skeleton are also discussed, according to bio-mimetic analogies (Fig. 5, bottom panel - see also Kapandji, 1970).

The final configuration is based on three artificial muscles - Wittenstein Motion Control Gmbh, link $\{1\}$ - placed in the forearm that move the three phalanges independently. The final design of two (of the 3 phalanges) is shown in Fig. 6 where the dimensions of the mechanical phalanges are similar anatomically to the human middle finger.

It is important to note that the "imitation" approach has recently stimulated further discussion (Schaal, 1999) and it has been adopted at the A.I. Laboratory of MIT \{2\} (development of a human-like finger system), at the LIRA-Lab - $\{3\}$ (the Babybot Project) 
and at the Humanoid Robotic Group Research at MIT - $\{4\}$ (see also the Biologically Inspired Robotics Laboratory - $\{5\})$.

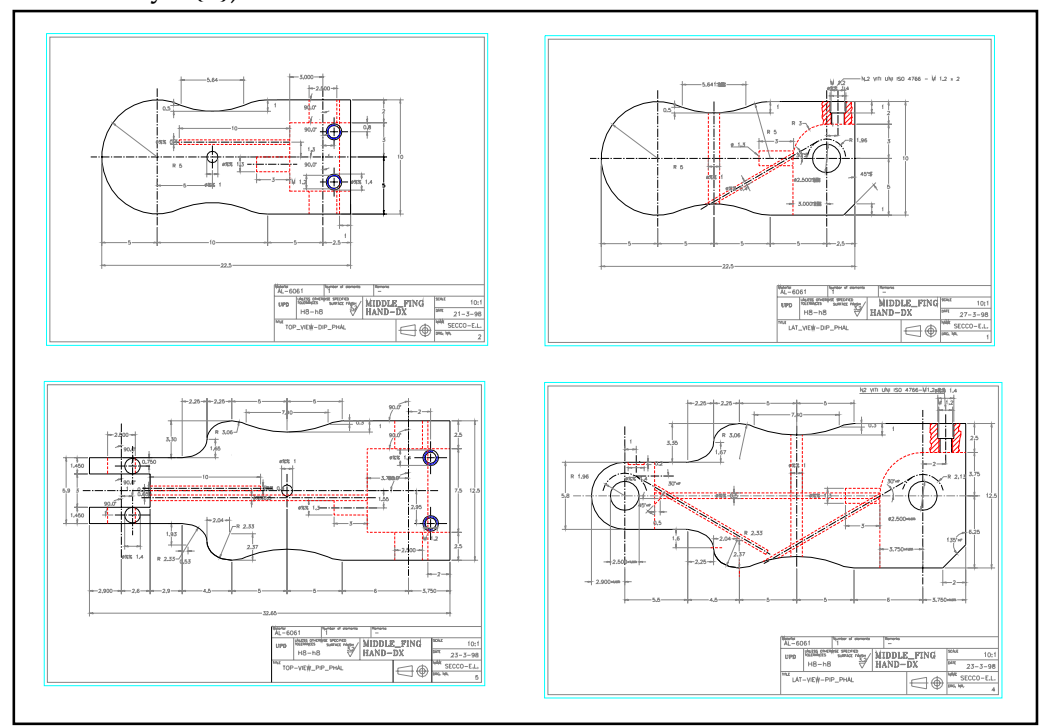

Fig. 6. Top and lateral view (respectively on the LEFT and on the RIGHT side) of the DIP and PIP phalanges (respectively on the TOP and on the BOTTOM). Quotes are expressed in $[\mathrm{mm}]$ : the mechanical components are dimensionally similar to the human phalanges morphology - from Secco, 1997.

\section{Bio-Mimetic Motion Planning}

We propose a method, based on both physiologic and engineering considerations, for the motion planning of a prosthetic finger. In particular, we exploit a minimum jerk approach to define the trajectory in Cartesian space. Then, cubic splines are adopted in the joint space. The redundancy problem arising from the presence of three links is solved by assuming that there is a constant ratio between the second and the third joint motion. The value of the proportional constant is determined by minimizing the maximum jerk in the joint space. It is found that this constant value can be suboptimally, but effectively, set to one for all the movements. This approach guarantees a natural movement of the finger as well as reduced vibrations in the mechanical structure and increased control performance.

\subsection{Introduction}

In the past few years there has been significant development of prosthetic hand devices and haptic perception. Many laboratories have produced multi dof artificial hands for robotic and/or prosthetic applications (see, e.g., Jacobsen et al., 1986; Rosheim, 1994; Melchiorri \& Vassura, 1995). In this context, after reproducing the morphology, it is also of interest to reproduce the natural movement of the fingers in order to perform different tasks, such as exploring an unknown object with an artificial hand (for teleoperation use) or reaching and handling an object with prosthetic devices (for the support of handicapped patients). However, this has to be done while also taking into account the design constraints of the mechanical structure and of the control architecture. 
From the physiological side, many studies have been published regarding the finger (Harris \& Rutledge, 1972; Buchner at al., 1988; Lee \& Rim, 1990; Hoff \& Arbib, 1993; Hahn et al, 1995; Brook et al., 1995), arm (Massone \& Bizzi, 1989; Okadome \& Honda, 1999), and human movements in general (Nelson, 1983; Plamondon, 1995ab), with both kinematic and dynamic approaches. In general, they conclude that natural movements are planned and executed by following optimization principles. In particular, we highlight the contribution of Flash \& Hogan (1985) who show that the upper limb movements in the proximal space are executed in order to minimize the square magnitude of the jerk (the derivative of the acceleration function) of the extremity in the Cartesian space over the entire movement. Following this result, Laczko et al. (2000) investigated, in multi-joint kinematic chains, the relative contribution of the velocities, accelerations, and jerks in the individual joints to the total endpoint jerk; they concluded that the term related to the individual joint jerk dominates over the others. From a robotic viewpoint, the problem of determining the motion of a multi joint finger has to face the redundancy of the system, i.e., there are an infinite number of joint configurations for a unique Cartesian fingertip position. In other words, the presence of the third joint/phalanx implies that we have three dof, namely one more than that necessary to address a motion in a two-dimensional plane. In general, the additional dof is exploited in order to minimize some objective function.

It is also recognized that the minimization of the joint jerk provides benefits to the mechanical structure of a robot manipulator, reducing the presence of vibrations and the joint wear and therefore increasing the robot life-span (Craig, 1989) as well as permitting the increase of trajectory control performances (Kyriakopoulos \& Saridis, 1988; Secco \& Magenes, 2002ab). In this context, different approaches to minimize the joint jerk in the trajectory planning of robot manipulators have been proposed in the literature (Simon, 1993; Piazzi \& Visioli, 2000)

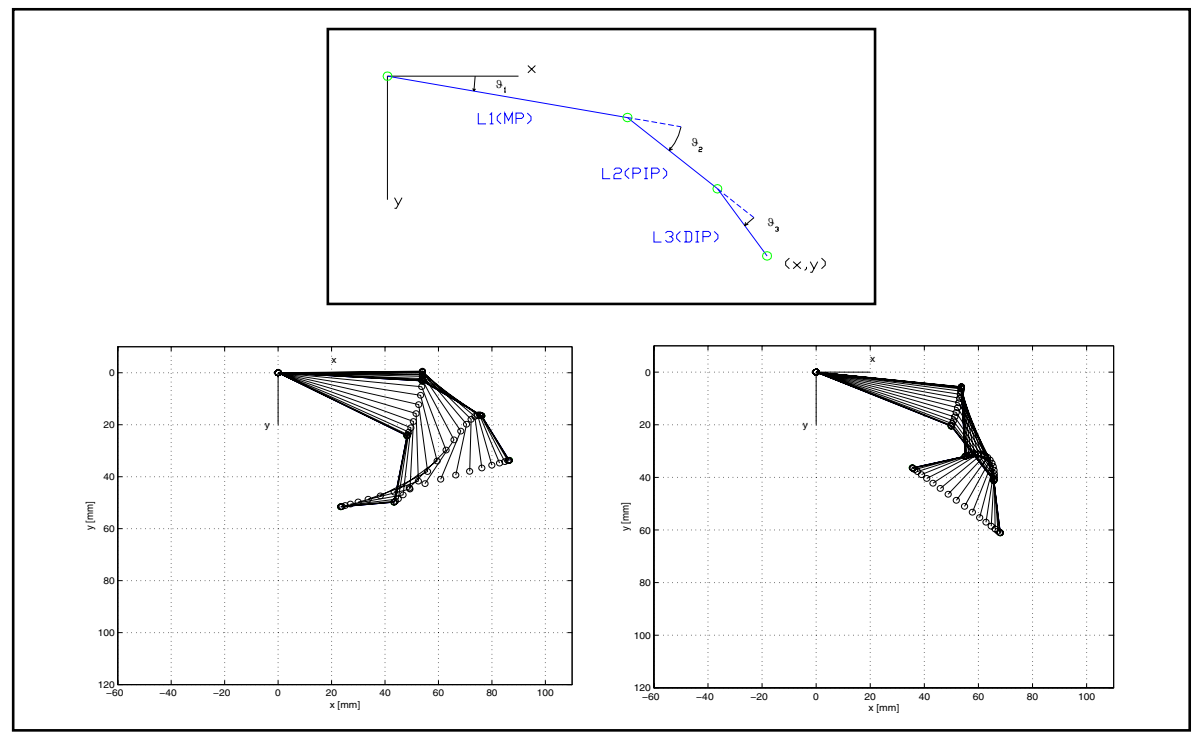

Fig. 7. TOP PANEL. Schematic representation of the prosthetic finger. BOTTOM PANEL. Evolution of 2 representative simulations of minimum jerk movements.

However, when the coordinated movement of a prosthetic arm, consisting of a multilink/multi-joint kinematic chain, has to be programmed and controlled in order to reach a 
determined position in the proximal space with the end-effector (the fingertip) for grasping, touching, or exploring an object, it seems useful to maintain a smooth motion of the fingertip along the straight line trajectory connecting its initial and final positions. This implies a smooth control of the fingertip in the Cartesian space, with null acceleration at the beginning and at the end of the motion. This should prevent accidental mechanical shocks of the fingertip on the object, due to possible minute errors in the joint actuators. Thus, in addition to physiological requirements, this represents an important mechanical feature, as a low impact is guaranteed in the fingertip approach to the object's surface.

Taking into account all these considerations, we propose a new approach for the motion planning of a prosthetic finger, based on the minimum-jerk principle. Basically, it consists of defining linear movements of the tip in the Cartesian space, defined in such a way to minimize the Cartesian jerk. Then, the inverse kinematic problem is solved by using cubic splines to interpolate the trajectory between the two known knots and by fixing a constant ratio between the second and the third joint angle. The value of the constant ratio is found by minimizing the maximum jerk of the three joints. The obtained results are consistent with physiological evaluations.

\subsection{The Minimum Jerk Principle}

\subsubsection{Motion Planning in the Cartesian Space}

We consider a human middle finger, shown in Fig. 7, where $L 1=54 \mathrm{~mm}, L 2=26 \mathrm{~mm}$, and $L 3=20 \mathrm{~mm}$ are the lengths of the phalanges ${ }^{1}$ and $\vartheta_{1}, \vartheta_{2}$, and $\vartheta_{3}$ are the MetacarpoPhalangeal (MP) joint, the Proximal-Inter-Phalangeal (PIP) joint, and the Distal-InterPhalangeal joint (DIP), respectively. From physiological considerations (Kapandji, 1970) it has been assumed that

$$
-30^{\circ} \leq \vartheta_{1} \leq 90^{\circ}, \quad 0^{\circ} \leq \vartheta_{i} \leq 90^{\circ}, i=2,3
$$

Only straight movements in the $X-Y$ plane are considered.

By applying the mathematical model developed by Flash and Hogan (1985) to the finger, it results that a natural movement from position $\left(x_{0}, y_{0}\right)$ to position $\left(x_{f}, y_{f}\right)$ starting at time $t_{0}=0$ and ending at time $t_{f}$ has to minimize the following function, (the time integral of the square of the magnitude of jerk):

$$
C=\frac{1}{2} \int_{0}^{t_{f}}\left[\left(\frac{d^{3} x}{d t^{3}}\right)^{2}+\left(\frac{d^{3} y}{d t^{3}}\right)^{2}\right] d t
$$

where $x$ and $y$ are the time-varying coordinates of the fingertip position. By solving the optimization problem, we see that the trajectory of the fingertip in the Cartesian space is uniquely determined as follows:

$$
\begin{aligned}
& x(t)=x_{0}+\left(x_{f}-x_{0}\right)\left[-6\left(\frac{t}{t_{f}}\right)^{5}+15\left(\frac{t}{t_{f}}\right)^{4}-10\left(\frac{t}{t_{f}}\right)^{3}\right] \\
& y(t)=y_{0}+\left(y_{f}-y_{0}\right)\left[-6\left(\frac{t}{t_{f}}\right)^{5}+15\left(\frac{t}{t_{f}}\right)^{4}-10\left(\frac{t}{t_{f}}\right)^{3}\right]
\end{aligned}
$$

\footnotetext{
${ }^{1}$ We applied this study also with the average American female middle finger $(L 1=42.11 \mathrm{~mm}, L 2=20.27$ $\mathrm{mm}$, and $L 3=15.60 \mathrm{~mm})$ and with the average American male middle finger $(L 1=46.22 \mathrm{~mm}, L 2=22.26$ $\mathrm{mm}$, and $L 3=17.12 \mathrm{~mm}$ - Garrett, 1971), obtaining the same results for all the three fingers. The length of the phalanges is not a critical issue as well (see details in Secco et al., 2002).
} 


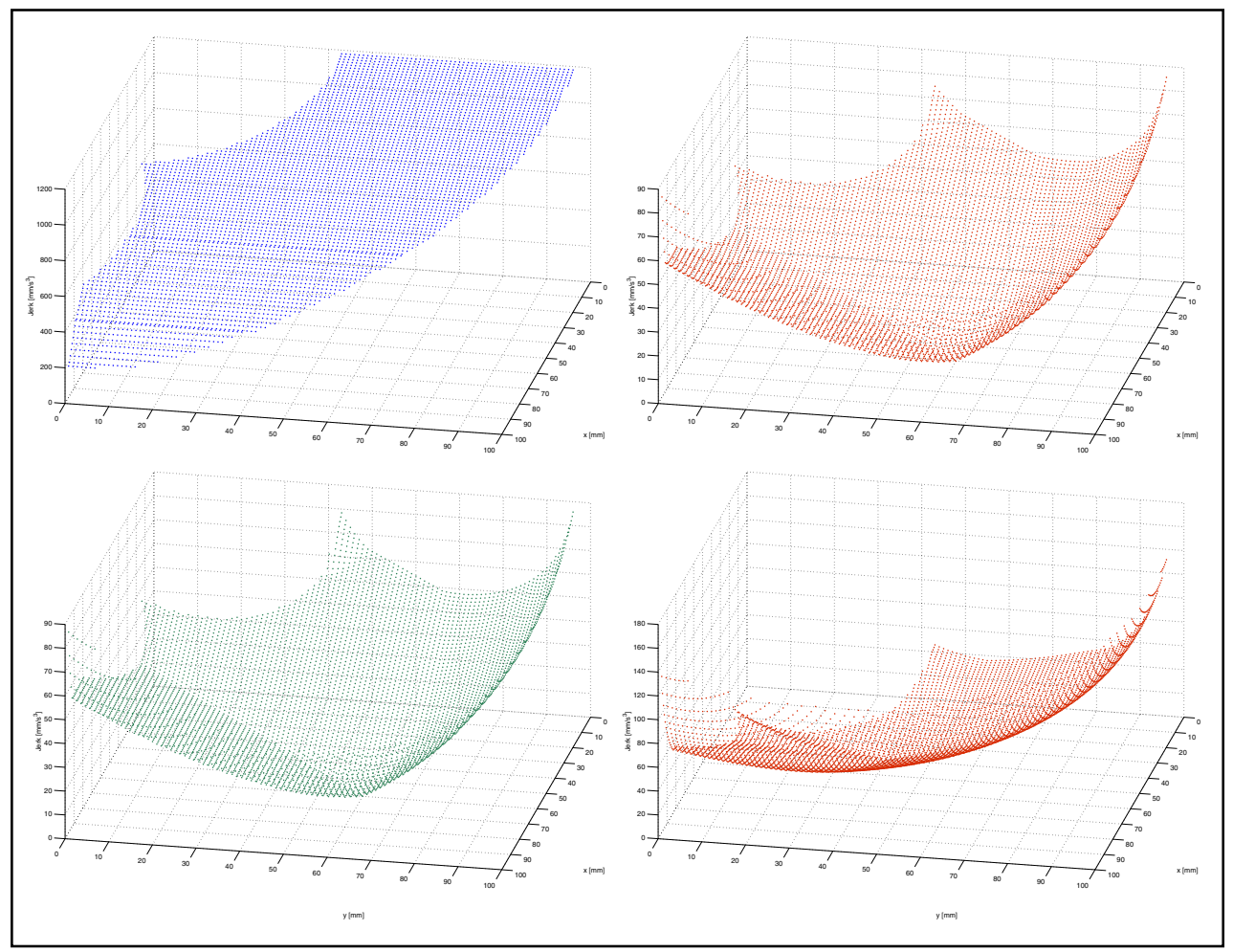

Fig. 8. Values of the maximum jerk (VMJ) obtained by using the value $K=1$ for motions starting from $(100,0)$ - TOP LEFT PANEL - and from $(60,60)$ - BOTTOM LEFT PANEL. VMJ by using the optimal value of $\mathrm{K}$ for motions starting from $(60,60)$ - TOP RIGHT PANEL and from $(20,60)$ - BOTTOM RIGHT PANEL.

\subsubsection{Motion Planning in the Joint Space}

Once the movement of the fingertip in the Cartesian space has been determined, the inverse kinematics problem has to be solved in order to calculate the motion law in the joint space, which is then applied to the robotic finger by using appropriate actuators. A practical solution, often adopted in industrial environments, is to select a sufficient number $m$ of equally spaced knots along the Cartesian trajectory.

For simplicity, we select the knots $d_{1}, \ldots, d m$, at time intervals equal to each other. Then, the corresponding joint configuration has to be determined by applying the inverse kinematics. In this context, the redundancy problem has to be faced. Namely, the presence of three joints causes that an infinite number of joint configurations solve the inverse kinematics problem. One of the simplest methods to effectively tackle the redundancy problem, consists of maintaining the angle of the third phalanx proportional to that of the second phalanx, namely,

$$
\vartheta_{3}=K \vartheta_{2}, K \geq 0
$$

Note that this assumption seems to be reasonable from a physiological point of view, since such proportionality - eq. (4) - drives the natural movment of the human finger (see, e.g., 
Harris \& Rutledge, 1972; Hahn et al., 1995; Secco, 2001). Then, for each joint, cubic splines (Craig, 1989; Lin et al., 1983) are adopted to interpolate the resulting displacements for each joint. The salient feature of the cubic splines is that they assure the continuity of the velocity and acceleration functions and, being of low order, they prevent large overshoots (see details in Secco et al., 2002).

\subsubsection{Optimization Problem}

In the framework proposed in the previous subsections, the resulting trajectory in the joint space depends on the design parameter $K$. An explicit solution of the inverse kinematic problem that depends on $K$ cannot be computed because of its high complexity. Therefore, the value of $K$ has to be fixed before the inverse kinematics and the cubic splines approach are applied. In this context, the inverse kinematic problem can be solved by applying a standard Newton-Raphson algorithm (Ortega \& Rheimboldt, 1970). Thus, once a value of $K$ has been selected, taking into account the physical constraints on the joint angles (1), the inverse kinematic problem can be uniquely solved.

An appropriate method to find the value of $K$ is to minimize the maximum jerk over the three joints. In other words, we have to solve the following constrained min max optimization problem:

$$
\min _{k \geq 0} \max \left\{\left|j_{k i}\right|: i=1, \ldots, n ; k=1,2,3\right\}
$$

subject to

$$
-30^{\circ} \leq \vartheta_{1} \leq 90^{\circ}, \quad 0^{\circ} \leq \vartheta_{i} \leq 90^{\circ}, \quad i=2,3 .
$$

From a practical point of view, an upper bound for the optimal value of $K$ can be easily found by taking into account again physiological consideration (Kapandji, 1970). A conservative upper bound $K^{+}=1.5$ has been selected, while, as a lower bound, the value $K^{-}=$ 0 has been retained. In order to find the optimal value $K^{*}$ that minimizes (5), a tight gridding (with step equal to 0.01 ) over the interval $\left[\mathrm{K}^{-}, \mathrm{K}^{+}\right]$has been performed, evaluating the maximum jerk for each value of $K$ and then selecting the optimal one (see details in Secco et al., 2002).

\subsection{Results}

The algorithm presented in Section 2.3 has been applied to a large number of movements, with different motion times (Secco et al., 2001). Here, for the sake of clarity, we focus on some significant results that illustrate the conclusions we draw. In particular, we consider straight movements performed in an interval time of $1 \mathrm{~s}$ (i.e., $t_{f}=1 \mathrm{~s}$ ) with $m=20$. As example, different configurations of the finger for particular movements are reported in Fig. 7.

First, we select all movements starting in $(100,0)$ - Fig. 7 - and ending in the points of the workspace. In all cases we obtain that the optimal jerk corresponds to a value of $K$ close to 1 . The resulting optimal jerk $j^{*}$ for each movement is shown in Fig. 8 - top left panel. Slightly different results are obtained for different starting points. Namely, the solution of the optimization problem does not always yield to the value $K=1$. In any case, it is of interest to compare the values of the jerk $j^{*}$ achieved for the optimal values of $K$ and the values of the jerk achieved by fixing $K=1$. They have been reported in Fig. 8 - top right panel and Fig. 8 bottom left panel, respectively, for movements starting in $(60,60)$ and in Fig. 8 - bottom right panel and Fig. 9 - left panel, respectively, for movements starting in $(20,60)$. 
It appears that the difference between the values of the maximum jerk achieved by selecting the optimal value of $K$ and $K=1$ is not very significant. This is confirmed by the fact that the resulting optimal value of $K$ is indeed $K=1$ for many movements.

The reader can appreciate what is affirmed through the illustrative example of Fig. 9 - right panel, where the final points of the movements (for the case $x_{0}=20$ and $y_{0}=60$ ) for which the optimal $K$ is equal to one are indicated. For a better evaluation of the results from an analytical point of view, the mean value of the maximum jerk and its standard deviation for the considered movements are reported in Table 1.
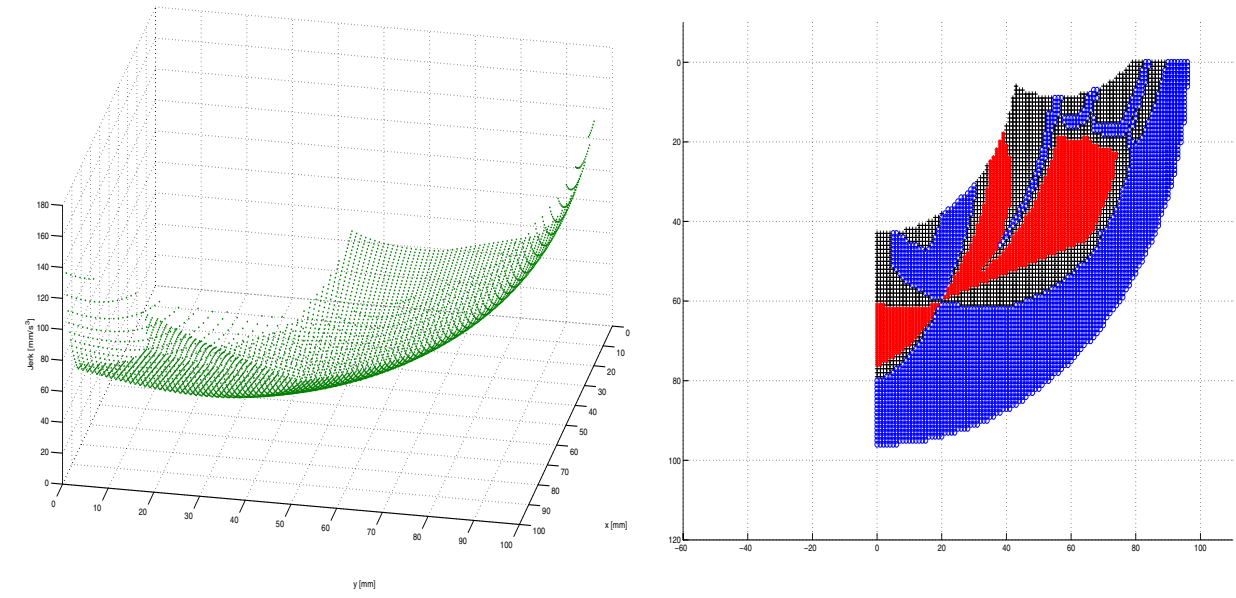

Fig. 9. LEFT PANEL. Values of the maximum jerk obtained by using the value $\mathrm{K}=1$ for motions starting from $(20,60)$. RIGHT PANEL. Final positions of motions starting from $(20,60)$ for which $K^{*}=1$ (indicated with blue " + "). Red dots (" $\circ$ ") represents the $K=1.5$ class, black cross $\left("{ }^{\prime \prime}\right)$ the uncertainty zone. The percentage range permitted to belong to each class is $10 \%$.

\subsection{Discussion}

The obtained results show that a suitable choice to solve the redundancy problem in the motion planning of the prosthetic finger consists of choosing a constant ratio equal to one between the PIP and the DIP joints. Although the proposed solution is theoretically suboptimal, from a practical point of view it is easy to implement and preserves the minimization of the maximum jerk in the joint space. In addition, the value $K=1$ is appropriate also from physiological considerations, as it reflects the behavior of a real human finger (Harris \& Rutledge, 1972; Secco et al., 2005; Sumbre at al., 2005).

It also worthnoting that for $K=1$ the inverse kinematic problem can be solved analytically (details in Secco et al., 2002).

Hence, the overall proposed methodology satisfies both physiological and engineering requirements as the planned movement reflects that of a human finger, both in Cartesian and in the joint space, and it ensures the prevention of vibrations and the increasing of the control performances by keeping the maximum jerk of the joints at a low level. 


\begin{tabular}{|c|cc|cc|}
\hline & \multicolumn{2}{|c|}{ Optimal K } & \multicolumn{2}{c|}{$\mathrm{K}=1$} \\
\hline Starting Point & mean & std & mean & std \\
\hline$(100,0)$ & 812.8 & 238.3 & 812.8 & 238.3 \\
\hline$(60,60)$ & 33.4 & 15.2 & 33.8 & 15.4 \\
\hline$(20,60)$ & 40.8 & 27.8 & 41.5 & 27.9 \\
\hline
\end{tabular}

Table 1. Mean value and standard deviation of the maximum jerk for different motions with the optimal value of $\mathrm{K}$ and with $\mathrm{K}=1$.

\subsection{Conclusions}

This strategy ensures both a smooth approach to the object along a straight line trajectory (with a low shock of the fingertip when touching the object) and low jerk at the joint level, as required by physiological and mechanical considerations. The redundancy robotic problem is solved by selecting a constant ratio (equal to one) between the PIP and the DIP joint, in order to minimize the maximum of the jerk functions of the three joints. This solution is suitable to be applied in a practical context since it satisfies engineering requirements (easiness of implementation, reduction of vibrations, increasing of the control performances, etc.) and at the same time it allows a physiological smooth natural movement.

\section{Bio-Mimetic Control: Teaching a Robot With Human Natural Movements}

After reproducing the morphology and the kinematics, we face a human inspired sensorimotor approach to robotic systems, to solve the problem of designing adaptive and learning robots for widely versatile tasks. Hence we present a $n n$ controller for driving the 3 dof finger to desired positions in space. The controller is taught with human movements of the corresponding finger. At the end of the training process, it is able to closely imitate the physiological control and the motion planning strategy of the human being. Generalization properties are shown after the training process (that is the capacity to move the device in different directions and places never seen during the teaching phase). This approach seems promising for controlling artificial prosthetic and robotic upper limbs in general.

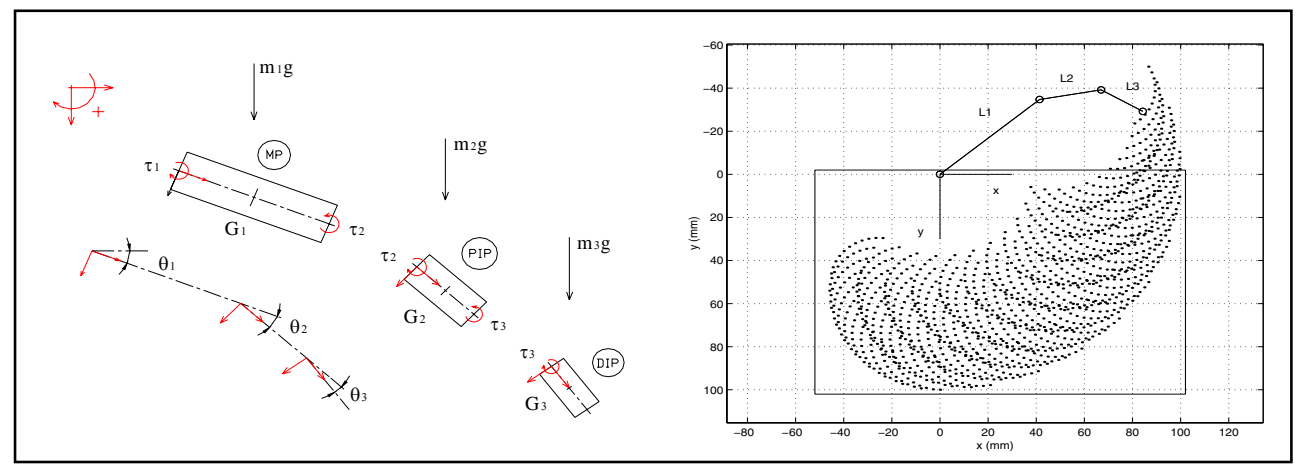

Fig. 10. LEFT PANEL. Conventions of the signs, the external forces and the motor torques applied to the three phalanges $-\tau_{1}, \tau_{2}$ and $\tau_{3}$ are the MP, PIP and DIP Phalanx torques. $G_{1}, G_{2}$ and $G_{3}$ are the 3 barycenters; $m_{1}, m_{2}, m_{3}, \vartheta_{1}, \vartheta_{2}, \vartheta_{3}$ are masses and angular displacements respectively - $g$ represents the gravitational contribute. RIGHT PANEL. Schematized representation of the geometrical model adopted for the middle finger in the vertical plane: the rectangular box represents the workspace domain of the nn controller. 


\subsection{Introduction}

The problem of designing and producing a humanoid robot, able to interact intelligently with the environment, has recently been inspired by the behavior-based approach that avoids high level cognitive representation and relies upon a tight connection between sensory and motor systems (Brooks \& Stein, 1994). Among the evident advantages that could derive from a human inspired sensorimotor approach to robotic systems are the reproduction of the outstanding fault tolerance, the adaptability to changed environments, the learning ability and the inherent versatility of human beings.

In this last paragraph we present a $n n$ controller for driving the 3 dof finger to a desired position in its proximal space. The controller is taught with human movements of the corresponding finger and, at the end of the training process, it is able to closely imitate the physiological control and the motion planning strategy in human beings.

Both for robotic and prosthetic applications, such control system driving multi dof artificial limbs in a smooth natural manner could represent a noticeable improvement. Moreover it is compulsory that the natural movement, instead of being controlled by overwhelming equations (see par. 2), can be executed in a straightforward manner and can be learned by examples from human experimental measurements. Thus, a possible methodological tool capable of achieving the desired specifications is represented by artificial $n n$. They allow us to setup the control system bypassing the computation of the explicit solution of the inverse dynamics problem.

The control law is obtained by optimizing the weights of the $n n$ through a supervised training procedure which utilizes a set of input-desired output pairs, taken from natural movements measurements.

\subsection{Methods}

The robotic finger is mechanically simplified as consisting of three links (the MP, PIP \& DIP phalanges) and three planar joints (the 3 articulations), on which three torques can be applied by means of small torque motors. A mechanical model of the artificial finger, a kinematic model of its movement and a dynamic model are developed. A similar approach could be easily readapted to the analysis and control of the upper limb too.

\subsubsection{The Plant and the Controller}

The mechanical properties of the finger have been previously characterized by the mass, the friction and the forces acting on each phalanx (Secco \& Magenes, 2002a). Each joint torque motor is provided by a rotational encoder giving the angular position and velocity of each phalanx with respect to the preceding one. The finger is acting on the vertical plane under the gravity action. Specifically we consider a planar model of the middle finger consisting of 3 rods (the 3 phalanges) and 3 hinges - the MP, PIP and DIP joints. The choice of neglecting lateral movement is supported both by the need of simplifying the problem and by the design constraints of a prosthetic/artificial middle finger. The finger configuration is defined by the three angles $\vartheta_{1}, \vartheta_{2}$ and $\vartheta_{3}$ as illustrated in Fig. 10 - according to the robotic Denavit-Hartenberg notation. The anatomical constraints and the dynamic phalanx properties are selected according to the anatomical behavior of the homologous human finger. These conditions univocally determine the workspace of the artificial fingertip $(x, y)$ $L_{1}, L_{2}$ and $L_{3}$ are defined as the MP, PIP and DIP phalanx lengths, $\vartheta_{1}, \vartheta_{2}$ and $\vartheta_{3}$ the respective relative angular dispositions. 
Whereas we need to check the behavior of the network by supposing a virtual movement and not using the real acquired movements, the adopted kinematic model follows a simple approach; we fix a smooth natural movement mathematically illustrated by the two conditions expressed in Eq. (2) \& (4) with $K=1$. The first equation comes from the minimum jerk theory which we are extending to the finger, while the second hypothesis stems from the necessity to support the first, because of the three dof redundancy (see the details in par. 2). By means of the imposed conditions we can describe a rectilinear and smooth movement of the fingertip (as reported on the background of Fig. 11 - top panel), providing an unimodal bell speed profile.

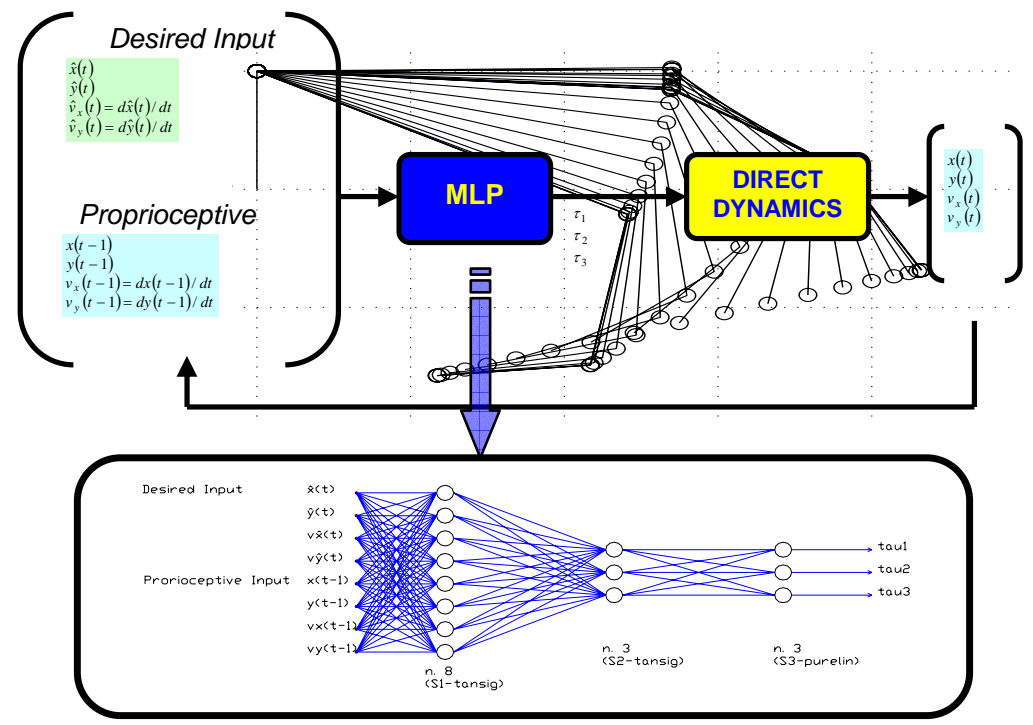

Fig. 11. TOP PANEL. The closed loop control system: the controller (MLP) is fed back by the proprioception of the muscle spindles. A direct dynamic block determines the effective movement due to the applied torques. BOTTOM PANEL. The Multi Layer Perceptron (MLP) nn: the structure of the I/O training vector, the activation functions and the number of neurons for each layer. In the configuration of the figure we have 8,3 and 3 neurons on the $1^{\text {st }}, 2^{\text {nd }}$, and $3^{\text {rd }}$ layer respectively. Activation functions are tansig, tansig and purelin respectively. Because of the type of activation functions, the three outputs are normalized between $[-1,1]$. Others structures were tested applying different architectures of the network with 3 and 4 layers and different numbers of neurons. The present configuration and the training set dimension are the results of an optimized compromise (see for details Secco \& Magenes, 2002a; see also Haykin, 1999).

The dynamics is governed by the kinematics. The kinematics is the real movement or the ideal movement that we have described above. In order to set up a dynamic model we define the properties of the artificial finger by establishing that a) the finger is moving on a vertical plane and it is subject to gravity, b) the phalanges are treated as homogeneous rods, c) the torques are provided by the motors positioned on the joints. Following Lagrange's approach we could write out the expressions of the three motor torques (Secco and Magenes, 2002ab). 
Finally, a Multi Layers Perceptron (MLP) $n n$ is setup. The input of the controller are the desired position and velocity of the fingertip (sensorial input) and the actual position and velocity (proprioceptive input). The output produces the joint torques. The MLP is fed back with the actual positions and velocities by closing the sensorimotor loop through the dynamics of the finger: the real movement due to the applied torques is sent back to update the proprioceptive input. The training performance is the minimization of the SSE (Sum Squared Error) of the output normalized torque. The three generalized forces are previously normalized between $[-1,1]$ - three factors of normalization are applied (one for each phalanx) - to be compatible with the domain of the $n n$ activation functions. The resulting performance function is:

$$
S S E=\sum_{i j k}\left(\tau_{i j k}-\hat{\tau}_{i j k}\right)^{2}
$$

where:

$\tau_{i j k}=$ simulated normalized value of the torque from the $n n$ output

$\hat{\tau}_{i j k}=$ desired normalized value of the torque from the dynamic equations

$i=1,2,3=$ number of phalanges

$j=1,2, \ldots, 7=$ number of movements

$k=1,2, \ldots, 23,24=$ number of steps/temporal passes for each movement

The adopted network has 8-5-3 neurons on the first, hidden and output layer respectively (Fig. 11 - bottom panel). Thanks to the dynamic model we are able to compute the necessary torque for each required movement and to train the controller. The learning procedure is back-propagation with adaptive learning rate and momentum term in batch mode 2 . Seven human movements are applied with the respective computed torque vectors for the learning phase

\subsubsection{Teaching the movement}

We performed some experimental measurements on the human movements of flexion and extension of the human finger. More precisely, we prepared an experimental setup to acquire the free flex-extension movements of the right index finger of human subject.

Before describing the apparatus, the measures and the results, we want to stress why it was important to perform this experimental phase.

First, the obtained data could be used to train the controller with actual natural movements. Through this procedure we could bypass the identification of the natural movement model. Moreover, we could get closer to the realization of a robotic/prosthetic device, which is trained by the observed movements (Da Cunha et al., 2000).

Second, the analysis of the data would have allowed us to improve/verify the kinematic model we apply to prepare the virtual movements (that is the kinematic model - Secco et al., 2002).

\footnotetext{
${ }^{2}$ Details on the structure's optimization and on the training procedure are reachable on Secco \& Magenes, 2002a.
} 
Third, we could control the effective torque patterns with respect to the training ones. We mean that we could apply the acquired movements to the artificial phalanges, deducing the consequent 'real' motor torques to move in this natural manner.

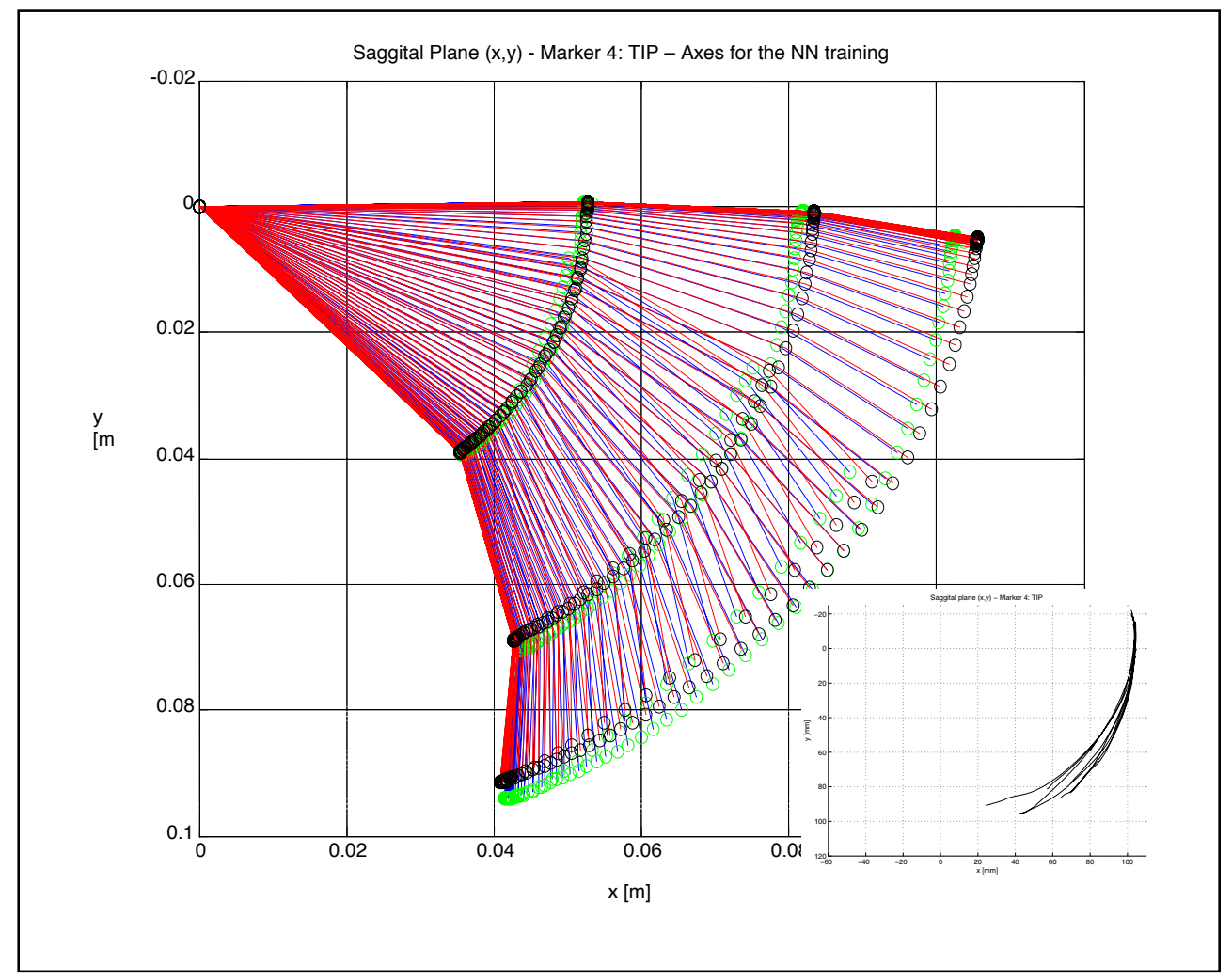

Fig. 12. MAIN PANEL. Finger path reconstruction during a partial flexion-partial extension movement and Fingertip (TIP) multiple path acquisitions - RIGHT BOTTOM PANEL.

A passive infrared markers system was used (Elite ${ }^{\circledR}$ System). We registered the movements by two cameras at a $100 \mathrm{~Hz}$ frequency. After the calibration and the estimation of the position error (less than 1\%), we applied 4 markers on the lateral side of the right index finger of 11 healthy volunteers. We acquired the movement on the saggital plane (we call it the $x y$ plane). We fixed the transducers to the MP articulation (Marker $\mathrm{n}$. 1), PIP articulation (Marker n. 2) and DIP (Marker n. 3) one. A fourth marker describing the Fingertip movement was fixed on a thimble that the volunteer was wearing during the acquisition. The palm was placed on a support, while the finger freely moved into the calibrated volume in front of the two cameras. The four markers were always visible from both the cameras. Approximately the finger moved on the $x y$ plane (apart from the movement of the palm on the hand support and the disturbances due to the movement of laterality - not inhibited). 
At the beginning the support was placed in such a manner that the palm distance from the flat was $50 \mathrm{~mm}$. In a second phase the support was rotated and the distance between the palm and the flat was increased to $66 \mathrm{~mm}$. For each phase and each person we asked to move the finger form a unique stretch limb configuration to a final fingertip position on the flat. The finger passed through the lighted band of a photocell: this was connected with a trigger that started the acquisition. Then the finger moved to one of the final possible positions on the flat: there were five possible final positions, equally spaced of $20 \mathrm{~mm}$ on a horizontal straigth line. In the best case, the total number of acquisitions for each of the 11 volunteers was 10 (5 movements with the support on the 'position $50 \mathrm{~mm}^{\prime}$ and other 5 movements in the 'position 66 $\left.m m^{\prime}\right)$.

We can describe a single training movement as a sequence of multiple micromovements. The controller structure (that is the $n n$ configuration and the training set dimension) was optimized for a 200 micro-movements set-up (Secco \& Magenes, 2002a; Haykin, 1999). Moreover we obtained not more than 8 movements of the same subject that were sufficiently extended in time. Thus we catched 7 of them for the training phase and one for the validation one. Considering the optimal number of micromovements was 200 and the number of entire available movements was 7 , then we divided each available acquisition in 24 successive positions of the fingertip at equal temporal intervals $\Delta t=30 \mathrm{~ms}$, from an initial fingertip position $\left(x_{i}, y_{i}\right)$ at time $t_{i}=0 \mathrm{~s}$ to a final one $\left(x_{f}, y_{f}\right)$ at time $t_{f}=30 \mathrm{~ms} * 24=0.72 \mathrm{~s}$. In this way 24 steps $/ \mu$-movements were used for each movement. The marker positions were previously filtered (low pass filter with a cut-off frequency of $10 \mathrm{~Hz}$ ). The markers positions were then corrected by imposing a constant length of the phalanges during the motion. The Cartesian fingertip velocity was computed, filtered with a smooth process and used to define the beginning of the movement. Then the resulted vector was resampled with a $30 \mathrm{~ms}$ period (Vicini, 2003). Then we computed the angular position, speed and acceleration of the three links to calculate the three torques for executing each $\mu$-movement. The torques were normalized and the entire set of vectors - containing the 7 movements - was setup for the training phase of the controller.. Finally a 200 thousand epochs training history performs the final error $S S E=0.8$.

\subsection{Results}

After training the MLP with the 7 real movements, we tested the same movements as input and we observed the normalized outputs. Here we show the results through graph bars and time patterns.

\subsubsection{Open Loop Control (i.e. no proprioception)}

For each movement, the SSE error of each temporal step is represented (24 temporal steps imply 24 torque values $\tau$, where $\tau(t)$ was applied for a moving finger from time $t-1$ to time $t$ ). The sum of the SSE of the three torque values (MP, PIP and DIP) for each pass is shown in the relevant bar (Fig. 13 - top left panel). The results are referring to the normalized torques. It is not manifested what happens to each phalanx torque in this representation. We need to look at each single phalanx torque computed by the $n n$ in the simulation phase, and to compare it with the expected value. Fig. 13 - top right panel refers to the temporal torque patterns of the training movement n. 5: theoretical torques are compared with simulation values 
obtained by the trained MLP. All the remaining 6 training movements show an equivalent behavior.
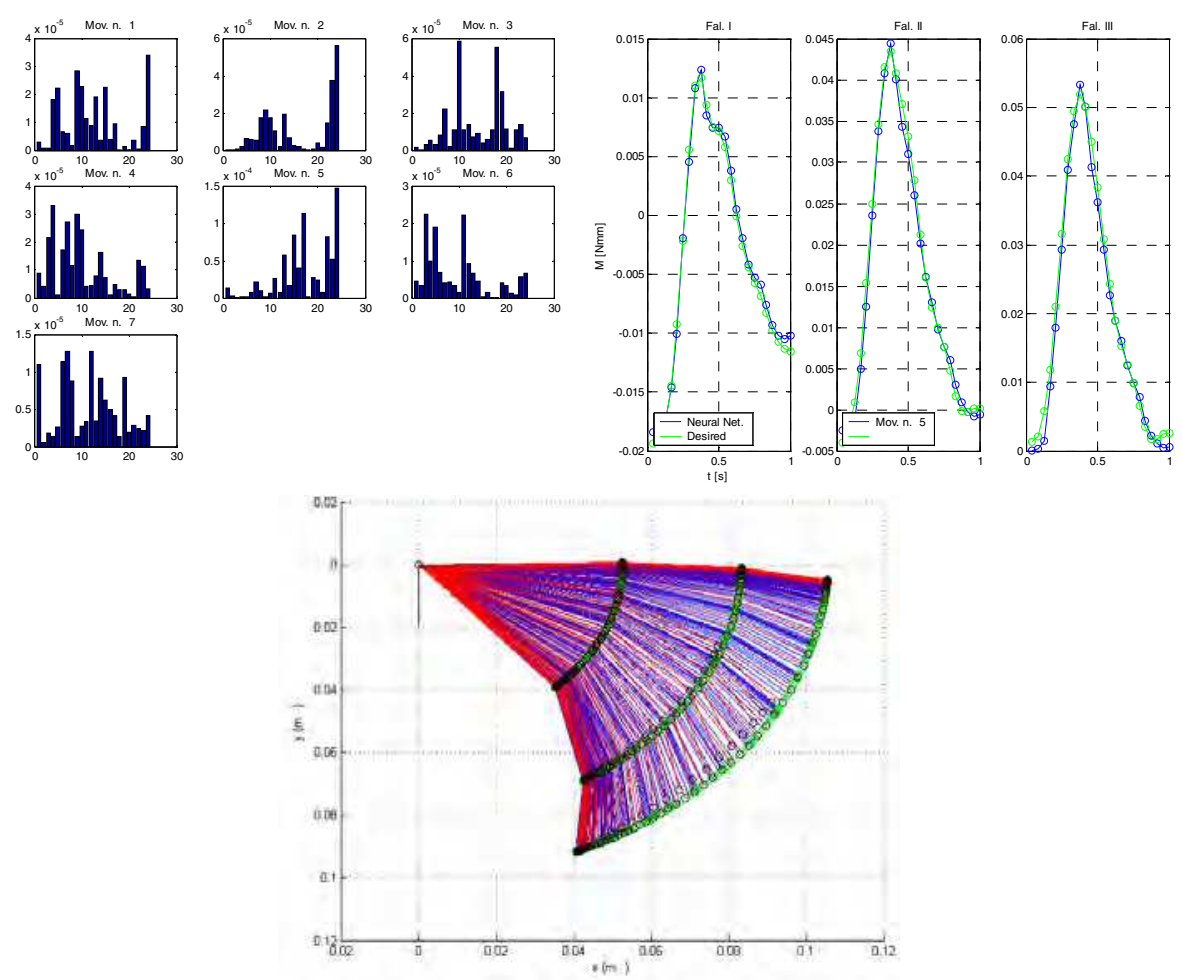

Fig. 13. TOP LEFT PANEL. The SSE of the normalized torques for each of the 7 training movements (step and $[\mathrm{N} \cdot \mathrm{m} / \mathrm{N} \cdot \mathrm{m}]^{2}$ ). Each bar represents the total SSE of the three torque values for each single step of the examined movements. TOP RIGHT PANEL. Course of the de-normalized torques of the training movement n. 5/7, '-' green solid lines are the desired/theoretical value, '-' blue solid lines represent nn simulation values. BOTTOM PANEL. We apply the integrator to the three patterns of motor torques. The torques are given as input to the integrator (the Direct Dynamic block of Fig. 11) as constant values on each interval of $30 \mathrm{~ms}$ for the entire execution time $(0.72$ s): the obtained final integrated movement, ' - ' blue lines, is compared with the real acquired one, '-' red lines.

It is possible to analyze and to represent the real/de-normalized torque values too, but we have to consider that:

- $\quad$ real de-normalized values do not give information about the success of the training phase: differences of SSE could be caused by different orders of magnitude of the real de-normalized phalanx torques values involved;

- de-normalized values trend is an homothetic transformation of the normalized ones because of the three applied factors of normalization (one for each phalanx). 
We applied the simulated and de-normalized torque to the Direct Dynamic block. The results give an answer about the effective precision of the $n n$ in executing the movement. We gave the generalization movement as input of this new configuration. We asked to the $n n$ to execute the $8^{\text {th }}$ never seen before movement. The graphical result is reported on Fig. 13 - bottom panel. It is compared with the real $8^{\text {th }}$ acquired movement. The two final fingertip positions are not coincident: the distance between the two positions (less than $10 \%$ with respect to the entire fingertip movement) comes from the cumulative error of the three simulated torques integration.

The final result depends on the integration of the highly non-linear dynamic equations. Every final trajectory of the training movements is well reproduced. Also the $n n$ seems to be able to simulate never seen movement during the training phase. Therefore we could conclude that all the training movements are re-executed by the MLP in a very satisfying manner and that the $n n$ can "implicitly learn" the dynamics of the system.

\subsubsection{Closed Loop Control (i.e. proprioceptive feedback)}

The dynamic control system was initially implemented as a sampled open-loop chain (feed-forward configuration). We did not closed the loop by up-dating the proprioceptive input during the motion. Actually, the feedback configuration of the controller represents the real natural condition. Thus we decided to realize the feedback procedure: we required the execution of a movement., then the movement was prepared and converted into a sequence of 24 different desired inputs and proprioceptive ones. For each step of motion from time $t-1$ to time $t$ (as usual, $t_{f}=0.72 \mathrm{~s}$, $t_{0}=0 \mathrm{~s}, \Delta t=30 \mathrm{~ms}$ ) a couple of inputs (desired + proprioceptive) was given; due to the input, the MLP computed the torques at time $t-1$ and time $t$. Interpolating the three values between $t-1$ and $t$, the three torques patterns were finally integrated. The new final fingertip position at time $t$ and its speed were fedback to update the proprioceptive input. This was the new state at time $t-1$ for the next step of motion from $t-1$ to $t$. The procedure was repeated until the $24^{\text {th }}$ step.

The same generalized movement was simulated by closing the loop (feedback configuration). The final trajectory is reported in Fig. 14 - top panel.

Finally, we wanted to verify the $n n$ ability of executing whatever movement by maintaing the same dynamics. We stressed the system by asking to execute a virtual rectilinear movement (as reported on Fig. 11 - top panel) by following the above defined kinematic model. We tested this in both the feed-forward and feed-back configuration. Graphical results are reported on Fig. 14 - bottom panel: we see that the feedback configuration (righ side of the mentioned figure) is clearly the best approach to imitate the biological control. We notice that both the curvilinear and the rectilinear movements are flexions. Extensions were tested with bad results.

However, even if the controller is not able to execute reverse (or not-forward) movements, it seems able to generalize quite well (also in terms of initial conditions). Considering that we could prepare more complete training sets (in terms of forward and reverse movements) with a consequent bigger capacity of moving in each direction, these results are very promising. Moreover, the application of such a system to the control of a device having a simpler dynamic - as a 2 dof arm - could be even more efficient. 


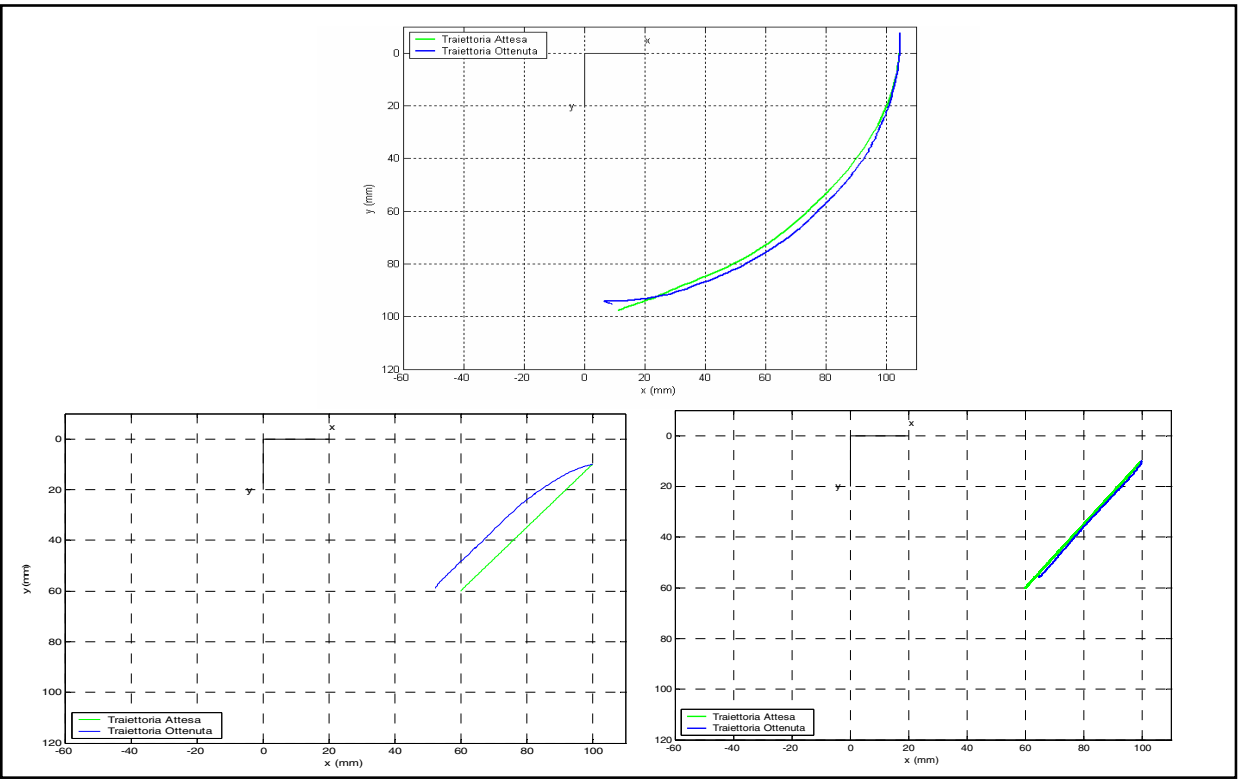

Fig. 14. TOP PANEL. The final fingertip real movement, '-' green line, is compared with the integrated one, '-' blue line, by closing the loop control chain. The 'in time' up-dating of the proprioceptive information is more visible in the final configuration. The trajectories are deduced by the integration of the three 'real' and simulated torques. BOTTOM PANEL. Fingertip 'real', '-'green line, and simulated, '-' blue line, trajectories obtained by the feedforward configuration controller (LEFT) and by the feedback configuration (RIGHT). The rectilinear movement is prepared by taking into account the kinematic model described in Par. 2, that is an extension of the minimum jerk theory (Flash \& Hogan, 1985) - details on (Secco et al., 2002).

\subsection{Discussion}

It has to be underlined that an optimization procedure must be carried out on the training set depending on the network structure. We mean that the next step could be to improve the number of Macro-Movements in the set of training examples, without reducing the number of $\mu$-Movements. This operation will require a re-modelling of the $n n$, due to the above optimization procedure (Haykin, 1999; Secco \& Magenes, 2002a).

A further step would be to define a performance parameter that is representative of the graphical results and of the final fingertip position. For instance the Euclidean distance between the desired final configuration and the obtained one could be a good proposal. Furthermore, it would be useful to define a performance index before the integration. In fact, despite the compensative and positive effect of the feedback, the torques computation of the $n n$ is controlled by the SSE, while the integration error is not 'controlled' yet. An intelligent solution could be to evaluate the area extension between the desired and obtained torques pattern. This is highly correlated with the integration. Finally, because of this correlation, we note (without the feedback) an increasing error on the fingertip position during the final part of the trajectory. An interesting development could be to train the 
network in function of a new Performance Parameter that depends on the integrated area between the MLP computed torques and the desired ones. However it has to be taken into account that the TIP reported trajectories represent the result of all the three torque integration, that is the final behaviour of a 3 dof mechanical chain. Due to the good results we would apply the same acquisition-learning-moving plant to the human arm, that could be initially treated as a more simple 2 dof planar limb.

The contents of such approach seem to have a high potential. By teaching the controller with few real movements, the system is able to 'generalize' and to learn the dynamics implicitly. We like imaging robots and/or prosthetic devices learning by the integration of different sensory information in a near future. A synergic fusion between proprioceptive and visual information has been proved a winning strategy. Further integration could be done by extending the application to the upper limb and, for what concerns the finger case, by involving tactile perception data (Caiti et al., 1995) in the device. The research trend on humanoid robots and/or helpful devices - prosthesis - has to be oriented towards this type of approach, that is the bio-mimetic approach - we mean imitating the biological system to build artifacts that better adapt to human needs (Magenes et al., 2002).

\section{References}

Ash, H. E. \& Unsworth, A. (1996). Proximal Interphalangeal Joint Dimensions for the Design of a Surface Replacement Prosthesis. Proc Instn Mech Engrs, 210, 95-108.

Ash, H. E. \& Unsworth, A. (2000). Design of a Surface Replacement Prosthesis for the Proximal Interphalangeal Joint. Proc Instn Mech Engrs, 214, 151-163.

Brook, N., Mizrahi, J., Shoham, M., Dayan, J. (1995). A biomechanical model of index finger dynamics. Med Eng Phys, 17, 54-63.

Brooks, R.A. \& Stein, L.A. (1994). Building brains for bodies. Autonomous Robots, 1 (1), 7-25.

Buchholz, B., Armstrong, T. J., Goldstein, S. A. (1992). Anthropometric Data for Describing the Kinematics of the Human Hand. Ergonomics, 35 (3), 261-273.

Buchholz, B. \& Armstrong, T. J. (1992). A Kinematic Model of the Human Hand to Evaluate Its Prehensile Capabilities. J. Biomechanics, 25 (2), 149-162.

Buchner, H.J., Hines, M.J., Hemami, H. (1988). A dynamic model for finger interphalangeal coordination, J Biomech, 21:(6), 459-468.

Caiti, A., Canepa, G., De Rossi, D., Germagnoli, F., Magenes, G., Parisini, T. (1995). Towards the realization of an artificial tactile system: fine-form discrimination by a tensorial tactile sensor array and neural inversion algorithms. IEEE SMC Transaction, 25 (6), $933-946$.

Da Cunha, F.L., Schneebeli, H.A., Dynnikov, V.I. (2000). Development of anthropomorphic upper limb prostheses with human-like interphalangian and interdigital couplings. Artificial Organs, 24 (3), 193-197.

Esteki, A. \& Mansour, J. M. (1997). A Dynamic Model of the Hand with Application in Functional Neuromuscular Stimulation. Annals of Biomedical Engineering, 25, 440-451.

Flash, T. \& Hogan, N. (1985). The coordination of arm movements: an experimentally confirmed mathematical model. J Neurosci, 5 (7), 1688-1703.

Garrett, J.W. (1971). The adult human hand: some anthropometric and biomechanical considerations. Human Factors, 13, 117-131.

Gupta, A., Rash, G.S., Somia, N.N., Wachowiak, M.P., Jones, J., Desoky, A. (1998). The Motion Path of the Digits. J. Hand Surg [Am], 23 (6), 1038-1042. 
Hahn, P., Krimmer, H., Hradetzky, A., Lanz, U. (1995). Quantitative analysis of the linkage between the interphalangeal joints of the index finger. J Hand Surgery, 20B, 696-699.

Harris, C. \& Rutledge, G.L. (1972). The functional anatomy of the extensor mechanism of the finger. J Bone Joint Surgery, 54 (1), 713-726.

Haykin, S. (1999). Neural Networks - A Comprehensive Foundation, Prentice Hall, $2^{\text {nd }}$ Ed

Hoff, B. \& Arbib, M. (1993). Models of trajectory formation and temporal interaction of reach and grasp. J Motor Behav, 25, 175-192.

Jacobsen, S., Knutti, D.F., Johnson, R.T., Biggers, K.B. (1986). Design of the UTAH/MIT dexterous hand. Proc IEEE Int Conf on Robotics and Automation, Raleigh NC, 15201532.

Kapandji, I. A. (1970). The Physiology of the Joints. E \& S Livingstone, Edinburgh \& London, $2^{\text {nd }} \mathrm{Ed}$, vol 1

Kyriakopoulos, K.J. \& Saridis, G.N. (1988). Minimum jerk path generation. Proc IEEE Int Conf on Robotics and Automation, 364-369, Philadelphia.

Laczko, J., Jaric, S., Tihanyi, J., Zatsiorsky, V.M., Latash, M. (2000). Components of the endeffector jerk during voluntary arm movements. J Appl Biomech, 16, 14-25.

Lee, J.W. \& Rim, K. (1990). Maximum finger force prediction using a planar simulation of the middle finger. Proc Instit Mech Eng, 204, 169-178.

Lin, C.-S., Chang, P.-R., Luh, J.Y.S. (1983). Formulation and optimization of cubic polynomial joint trajectories for industrial robots. IEEE Trans Autom Control, AC-23 (12), 1066-1074.

Magenes, G., Ramat, S., Secco, E.L. (2002). Life-like sensorimotor control: from biological systems to artifacts. Current Psychology of Cognition, 21 (4-5), 565-596.

Massone, L. \& Bizzi, E. (1989). A neural network model for limb trajectory formation. Biol Cybern, 61, 417- 425.

Melchiorri, C. \& Vassura, G. (1995). Implementation of whole hand manipulation capability in the U.B. Hand system design. J Adv Robot, 9 (5), 547-560.

Nelson, L.W. (1983). Physical principles for economies of skilled movements. Biol Cybern, 46, 135-147.

Okadome, T. \& Honda, M. (1999). Kinematic construction on the trajectory of sequential arm movements. Biol Cybern, 80, 157-169.

Ortega, J.M. \& Rheimboldt, W.C. (1970). Iterative solution of nonlinear equations in several variables, Academic Press, New York.

Pheasant, S. (1996). Bodyspace: Anthropometry, Ergonomics and the Design of Work, Taylor and Francis, London. 2nd Edition.

Piazzi, A. \& Visioli, A. (2000). Global minimum-jerk trajectory planning of robot manipulators. IEEE Trans Ind Electron, 47 (1), 140-149.

Plamondon, R. (1995a). A kinematic theory of rapid human movements: part I. Movement representation and generation. Biol Cybern, 72, 295-307.

Plamondon, R. (1995b). A kinematic theory of rapid human movements: part II. Movement time and control. Biol Cybern, 72, 309-320.

Rosheim, M.E. (1994). Robot evolution, Wiley, New York.

Craig, J.J. (1989). Introduction to robotics: mechanics and control, Addison-Wesley, $2^{\text {nd }}$ Ed, New York.

Schaal, S. (1999). Is imitation learning the route to humanoid robots? Trends in Cognitive Sciences, 3 (6), 233-242. 
Secco, E.L. (1997). Studi per la Realizzazione di una Mano Artificiale: Progettazione di un Dito a 3 gdl Ottimizzato per la Funzione di Presa. Master Thesis, University of Padova, Italy.

Secco, E.L., (2001). Movement Control of a 3 d.o.f. artificial finger: dynamic learning and execution of the natural movement. PhD Thesis, University of Pavia, Italy.

Secco, E.L., Visioli, A., Magenes, G. (2001). A Minimum Jerk Approach for the Motion Planning of a Prosthetic Finger. Proc. International Society of Biomechanics, Zurich.

Secco, E.L. \& Magenes, G. (2002a). A feedforward neural network controlling the movement of a 3 degree of freedom finger. IEEE SMC Transaction Part A, 32 (3), 437-445.

Secco, E.L., Magenes, G. (2002b). A Life-Like Control Algorithm for the Natural Movement of a 3 dof Finger. Proc. Int. Joint Conf. BMES EMBS, 2375-2376, Houston.

Secco, E.L., Visioli, A., Magenes, G. (2002). Minimum jerk motion planning for a prosthetic finger. Journal of Robotic Systems, 21(7), 361-368.

Secco, E.L., Valandro, L., Caimmi, R., Magenes, G., Salvato, B. (2005). Optimization of TwoJoint Arm Movements: A Model Technique or a Result of Natural Selection ?. Biological Cybernetics, 93 (4), 288-306.

Simon, D. (1993). The application of neural networks to optimal robot trajectory planning. Robot Auton Syst, 11, 23-34.

Sumbre, G., Fiorito, G., Flash, T., Hochner, B. (2005). Motor Control of Flexible Octopus Arms. Nature, 433, 595.

Vicini, O. (2003). Controllo neurale di un dito robotico allenato tramite movimenti di un dito umano. Master Thesis, University of Pavia, Italy.

\section{Links}

\{1\} Wittenstein Motion Control Gmbh http://www.wittenstein.de/

$\{2\}$ J. Lauren Banks http://www.ai.mit.edu/people/jessical

\{3\} The Babybot Project, LIRA-lab http://www.lira.dist.unige.it/Projects/Projects/Babybot/Baby_bot.htm

\{4\} The Humanoid Robotic Group http:/ /www.ai.mit.edu/projects/humanoid-robotics-group/

$\{5\}$ The Bio-Robots http:/ / biorobots.cwru.edu/

\section{Acknowledgment}

We would like to thank professor Antonio Visioli for scientific advice. 


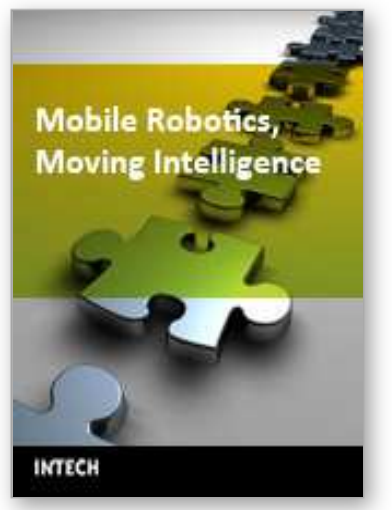

\author{
Mobile Robotics, Moving Intelligence \\ Edited by Jonas Buchli
}

ISBN 3-86611-284-X

Hard cover, 586 pages

Publisher Pro Literatur Verlag, Germany / ARS, Austria

Published online 01, December, 2006

Published in print edition December, 2006

This book covers many aspects of the exciting research in mobile robotics. It deals with different aspects of the control problem, especially also under uncertainty and faults. Mechanical design issues are discussed along with new sensor and actuator concepts. Games like soccer are a good example which comprise many of the aforementioned challenges in a single comprehensive and in the same time entertaining framework. Thus, the book comprises contributions dealing with aspects of the Robotcup competition. The reader will get a feel how the problems cover virtually all engineering disciplines ranging from theoretical research to very application specific work. In addition interesting problems for physics and mathematics arises out of such research. We hope this book will be an inspiring source of knowledge and ideas, stimulating further research in this exciting field. The promises and possible benefits of such efforts are manifold, they range from new transportation systems, intelligent cars to flexible assistants in factories and construction sites, over service robot which assist and support us in daily live, all the way to the possibility for efficient help for impaired and advances in prosthetics.

\title{
How to reference
}

In order to correctly reference this scholarly work, feel free to copy and paste the following:

Emanuele Lindo Secco and Giovanni Magenes (2006). Bio-Mimetic Finger: Human Like Morphology, Control \& Motion Planning for Intelligent Robot \& Prosthesis, Mobile Robotics, Moving Intelligence, Jonas Buchli (Ed.), ISBN: 3-86611-284-X, InTech, Available from:

http://www.intechopen.com/books/mobile_robotics_moving_intelligence/biomimetic_finger_human_like_morphology_control_amp_motion_planning_for_intelligent_robot_amp_

\section{INTECH}

open science | open minds

InTech Europe

University Campus STeP Ri

Slavka Krautzeka 83/A

51000 Rijeka, Croatia

Phone: +385 (51) 770447

Fax: +385 (51) 686166

www.intechopen.com
InTech China

Unit 405, Office Block, Hotel Equatorial Shanghai

No.65, Yan An Road (West), Shanghai, 200040, China

中国上海市延安西路 65 号上海国际贵都大饭店办公楼 405 单元

Phone: +86-21-62489820

Fax: $+86-21-62489821$ 
(C) 2006 The Author(s). Licensee IntechOpen. This chapter is distributed under the terms of the Creative Commons Attribution-NonCommercialShareAlike-3.0 License, which permits use, distribution and reproduction for non-commercial purposes, provided the original is properly cited and derivative works building on this content are distributed under the same license. 